Journal of Counselor Practice, 11(1):1-33, 2020

DOI: 10.22229/afa1112020

Copyright (C) Ohio Counseling Association

\title{
African Americans' Perceptions of Mental Illness and Preferences for Treatment
}

\author{
Janeé R. Avent Harris ${ }^{1}$ \\ East Carolina University \\ Loni Crumb \\ East Carolina University \\ Allison Crowe \\ East Carolina University
Jasmine Garland McKinney
East Carolina University

Historically, African Americans have lower rates of help seeking as negative perceptions of mental illness and treatment have persisted. Previous researchers identified stigma as an important factor in these attitudes and behaviors. In this current study, 210 African American participants responded to open-ended survey questions assessing these topics. We conducted a qualitative content analysis to identify themes. The themes that emerged included: 1) Stigma: Feeling ashamed and being shamed, (2) Violenceldanger, (3) Perceptions of ability, (4) Indicator of weakness, (5) Dismissing mental health issues, and (6) Religion and spirituality.

Keywords: African Americans, mental health, stigma, perceptions, treatment

African Americans use mental health services at about half the rate of Caucasian and Asian Americans (Substance Abuse and Mental Health Services Administration,

\footnotetext{
1 Correspondence may be sent to: Janeé R. Avent Harris, Department of Interdisciplinary Professions (IDP), East Carolina University (ECU), Ragsdale Hall 225A Mail Stop 121, Greenville, NC 27858, aventj16@ecu.edu, 252-737-1255.
} 
SAMHSA, 2015); however, in many instances, African Americans are at higher risk for mental illness. The Centers for Disease Control (CDC, 2016) reports that African Americans/Blacks have higher rates (3.4\% of adults) of severe psychological distress compared to Non-Hispanic Whites (3.2\% of Whites). Specifically, depression, anxiety, and post-traumatic disorder are common among African Americans (Hall; 2018; SAMSHA, 2015; Office of Minority Health, 2016). Reasons for this can include economic, social, educational, and physical health challenges that are present in Black communities (Singh et al., 2017). Given the disparities in help seeking and the proclivity for African Americans to experience mental illness, it is important to explore their attitudes towards mental illness and treatment.

\section{African American's Beliefs about Mental Health, Treatment, and Culture}

Researchers have explored the topic of the treatment gap (defined as the difference between those who need mental health services and those who seek mental health services), in order to understand what factors might impact it, particularly among racial and ethnic minorities (Authors 2016, 2019; Dua et al., 2011; McGuire \& Miranda, 2008; Wong et al., 2017). Mental health related stigma is consistently identified as a deterrent to help-seeking (Fripp \& Carlson, 2017), and those who carry these negative attitudes towards seeking help are less likely to seek treatment (Authors, 2019; Fripp \& Carlson, 2017). The following is a summary of the existing literature that explores this topic among African American adults. We describe research on stigma, coping, and how these attitudes impact help seeking behaviors. 
In a study of 201 African American adults (Sanders Thompson, Brazile, \& Akbar, 2004), participants identified several barriers to help-seeking including cultural norms, stigma, financial constraints, lack of knowledge, and preference for alternative resources (e.g., the church) rather than formal mental health services. Distrust, fears of misdiagnosis, labeling, and brainwashing also surfaced. In addition to these challenges, Matthews, Corrigan, Smith, and Aranda (2006) discussed culturally determined coping styles that impact attitudes and help-seeking behaviors of African American adults. First, self-reliance or the notion that a person should not look to others for support but rather deal with the stress, hardship, or emotional pain independently instead of reaching to others for informal or formal assistance.

In addition to self-reliance, African American adults also have vocalized that their self-images such as the "strong black woman" (Matthews at al., 2006, p. 258), do not coincide with seeking support when stressors arise. Participants described strong mental health, poor mental health, and severe mental illness. A list was generated for each and words included - stable, ability to cope (for good mental health), withdrawn, stressed, weird behavior (mental health problems), and dangerous, violent, crazy (to describe serious mental illness). When asked about the causes of mental illness, participants mentioned psychological (trauma or stress), biological (chemical imbalance), sociological (racism, oppression), spiritual (not being right with God), and supernatural (possessed by a demon). Few focus group members viewed mental illness as a treatable condition, and the people who understood mental illness as treatable linked this to religious intervention (anything is possible with God). Most of the participants (with the exception of the mental health 
providers) did not express a clear belief in mental health professionals as having a positive impact on the recovery process for a mental health concern.

Culturally determined coping styles and strategies were strongly endorsed. Five themes emerged and included: self-reliance (be strong and bear with it), self-images that are culturally determined (the strong black woman), the importance of religion and spirituality as coping (the Lord will take care of the problem), the use of informal support systems (staying within the family), and prevalence of unhealthy coping strategies among African Americans (alcohol and other drugs). Lack of awareness, costs, cultural beliefs about mental illness and treatment (negative beliefs about medication, mistrust), as well as mental illness stigma were discussed. Determinants of mental illness stigma included beliefs from older generations being passed down, a lack of information about symptoms and signs of a mental illness. One of the conclusions from the authors was that African Americans certainly hold some culturally-based biases that inhibit help seeking, and that future studies might examine attitudes from other racial ethnic groups to see how these compare.

The importance of religion and spirituality as coping has also been named as a reason many African Americans do not seek formal mental health services (Avent, Cashwell, \& Brown-Jeffy, 2015; Avent Harris \& Wong, 2018; Hays \& Lincoln, 2017), as the Black church has been used to cope with a mental health concern, or as a reason to not discuss the mental health issue at all, and that "the Lord will take care of it" (Matthews at al., 2006, p.258). Informal support systems, such as family and friends is another means of coping among African Americans, as many feel more comfortable discussing struggles in these more intimate settings as opposed to formal ones (Chatters, Taylor, Lincoln, \& 
Schrorpfer, 2002). Additionally, participants in Matthew et al. (2006) discussed habits such as substance use or overeating as examples of common, unhealthy coping behaviors that can replace mental health services.

In addition to alternative coping methods (Matthews et al., 2006), lack of awareness about available community mental health resources, high costs associated with mental health services, cultural beliefs about mental illness and treatment (negative beliefs about medication, mistrust), and mental illness stigma are factors that impact help-seeking in African Americans. Rather than risk having a negative experience with a professional from a majority group, African Americans might seek solace from each other and their church communities (Avent Harris \& Wong, 2018; Dempsey et al., 2016; Keating \& Robertson, 2004; Lincoln \& Mamiya, 1990). These empirical studies highlight the culturally nuanced perspectives African Americans hold that may inhibit help seeking. Although the relationship between the African American church and mental health concerns has been explored recently, the last study that specifically looked at attitudes and help-seeking behaviors of African American adults was conducted well over ten years ago so this research will add to the current state of knowledge on this topic.

Stigma, stereotypes, and marginalization. In order to explore such attitudes and preferences for mental health treatment, it is necessary to consider mental health stigma for African Americans within a broader cultural context. Many ethnic/racial minorities already face stereotypes and prejudices (Keating \& Robertson, 2004). To be open about mental health issues would make one vulnerable to further labeling, marginalization, and oppression (Gary, 2009). Anglin, Link, and Phelan (2006) found that African Americans 
were more likely than Caucasian participants to believe that someone with schizophrenia or major depression would act violently; however, African American participants were less likely to believe that the individual should be blamed or punished for violent behavior. So, although there were racial differences in stigmatizing attitudes, these negative perceptions did not translate into endorsing harsher treatment or blame.

Perceived dangerousness. To extend the line of inquiry into attitudes from various racial and ethnic groups, a follow up study (Anglin, Link, \& Phelan, 2006) looked at how a nationally representative group of African American participants compared to Caucasian participants in attitudes towards someone with a mental illness using vignettes. Of particular question was how participants might view a character in the vignette as dangerous and related attitudes. They noted that if African Americans tended to view people with mental illness as more prone to violence then Caucasians, then perhaps African Americans will assign more blame and responsibility and have rejecting attitudes and punishing behaviors. In the vignette, a person with a mental illness was described, and three questions assessed perceived dangerousness, blame, and punishment. Eighty-one African Americans and 590 Caucasian participants responded to questions and results indicated that African Americans were more likely than the Caucasian participants to believe that someone with schizophrenia or major depression would act violently. African American participants were less likely to believe that the individual should be blamed or punished for violent behavior. So, although there were racial differences in stigmatizing attitudes (dangerousness) these negative perceptions did not translate into endorsing harsher treatment or blame. Thus, attitudes are complex as they relate to mental health 
concerns, but the consideration of racial differences seems important and worthy of further investigation.

In 2007 authors (Rao, Feinglass, \& Corrigan) examined whether racial and ethnic differences existed in stigmatizing attitudes towards those with mental health concerns among community college students. Perceived dangerousness and desire for segregation from a person with a mental illness before and after an anti-stigma intervention was of particular attention. Before the anti-stigma intervention, African Americans and Asians perceived those with mental illness as more dangerous and desired more segregation than Caucasians. Latinos perceived people with a mental illness as less dangerous and desired less segregation than Caucasians. After the anti-stigma intervention, the same patterns emerged with the exception of Asian participants, who had a significant change and perceived those with mental illness as least dangerous of all the racial ethnic groups. The authors concluded that racial and ethnic background may in fact shape stigma and that antistigma intervention might be effective if they are targeted to particular racial and ethnic groups.

Beliefs about coping with a mental illness. Ward and Heidrich (2009) explored African American women's beliefs about mental illness and coping behaviors, stigma and help seeking, relationships between these, and age differences in these variables. The sample included 185 African American women between the ages of 25 and 85 who completed a survey on these topics. Results suggested that women believed that mental illness was caused by several factors (family stress, social stress due to racism), that mental illness is a cyclical in nature, and that there are serious consequences from a mental health 
concern but that it can be controlled by treatment. Low perceptions of stigma were found, and coping strategies included prayer, faith, informal supports, seeking medical care, and seeking mental health care. Related to differences according to age group, differences were found in all categories except stigma. Middle aged and older women were significantly more likely to believe alcohol and other drugs caused mental illness compared to other women. Older women were significantly more likely to indicate they would seek treatment than both younger and middle-aged women and less likely to use avoidant coping than younger and middle aged women. There were no significant differences related to stigma in the age groups. The authors mentioned that the results related to low levels of stigma in the African American sample were contrary to previous studies on this topic. Another implication put forth by the authors related to coping using faith and religion, and that more research related to religious coping, mental illness, and help seeking behaviors is warranted. Thus, attitudes are complex as they relate to mental health concerns, but the consideration of current racial differences is worthy of further investigation.

\section{Purpose of the Study}

Gary (2009) called for researchers from across disciplines to study how stigma impacts the help-seeking behaviors of ethnic/racial minorities. Previous researchers have conducted qualitative (e.g., Avent et al., 2015; Avent \& Wong, 2018) and quantitative studies (e.g., Fripp \& Carlson, 2017) to gain a deeper understanding about African Americans' perceptions of mental illness and preferences for treatment. Participant samples in existing qualitative studies tend to be small and more homogenous (e.g., Avent 
et al., 2015; Avent \& Wong, 2018). Fripp and Carlson (2017) suggested: "by adding a qualitative component, future researchers can ask questions regarding patterns of thinking toward mental health services (p. 90)." The emergent nature of qualitative research fits, particularly for populations who may have been historically underserved, oppressed, and marginalized (Hays \& Singh, 2012). Therefore, the purpose of this study was to add to the existing literature, by surveying a larger, more diverse group of African Americans, with an overall aim to further understand perceptions of mental health within their communities as well as their preferences for seeking help with emotional and mental concerns. The following research question(s) guided the current study: How do African Americans perceive stigma to impact mental health and help-seeking? What are the attitudes toward mental illness, and help-seeking within the African American community?

\section{Method}

The study received University and Medical Center Institutional Review Board approval at the authors' university. We used a qualitative content analysis research method. Qualitative content analysis is a method used for the subjective interpretation of the content of text data through a systematic data reduction and data classification process (Elo \& Kyngas, 2007). Qualitative content analysis entails coding and identifying themes or patterns (Hsieh \& Shannon, 2005) which enables researchers to understand social processes to provide knowledge, new insights, and a representation of facts in a scientific manner (Zhang \& Wildemuth, 2009). The research questions, exploratory nature of the subject matter, and focus on the African American culture made qualitative inquiry the best methodological approach for this study (Banyard \& Miller, 1998; Hays \& Singh, 2012). 


\section{Theoretical Framework}

The theoretical framework that guides this study is Critical Race Theory (CRT; Bell, 1995). Theoretical frameworks are an important part of qualitative research as they provide additional clarity in examining and understanding a particular phenomenon (Collins \& Stockton, 2018). CRT theorists posit that one must assess society and then radically change it. CRT considers the ways in which systemic forces impact marginalized communities' experiences. This disposition counters narratives that blame African Americans and focus on a deficit perspective. This theory is relevant to this particular study because of its focus on African Americans, who are often underrepresented in counseling services. Rather than focusing solely on African American help-seeking behaviors from a blame perspective it is more culturally responsible to seek understanding and consider the ways race and racism shape perceptions and ideas about mental illness (Bell, 1995).

\section{Research Team}

Our research team included two African American women and one European American woman all with previous experience conducting qualitative research. We participated in bracketing exercises throughout the research process. Bracketing is a process in which researchers acknowledge and temporarily set aside past experiences, assumptions, and potential biases that may influence the data and research process (Hays \& Singh, 2012). Each researcher completed bracketing exercises in a reflexivity journal when conceptualizing the study, during data collection and data analysis, and while writing 
the results. One of the major themes from the bracketing exercises was the idea that external and internalized messages would impact African Americans who experience stigma and their attitudes towards mental health. As the study progressed, many of the identified findings confirmed our initial thoughts. We were alarmed to see the prevalence of the violence/dangerous theme in the data and met as a group to discuss our reactions to the data throughout the research process.

\section{Procedure and Participants}

Participants were recruited from the general population across the United States (U.S.). We used a cluster sampling procedure (Creswell \& Cresswell, 2018) via Qualtrics Panel. A recruitment email was distributed with a link to an electronic survey to explore the relationship between self-stigma, public stigma, attitudes toward mental health treatment, and positive and negative affect. A total of 2,749 respondents received the electronic survey and 632 completed it, for a response rate of approximately $23 \%$. Participants who completed the survey received an incentive (e.g., points towards a gift card). Part one of the electronic survey included demographic questions (e.g., age, race).

Part Two of the electronic survey included six quantitative measures (i.e., the Perceived Stress Scale, Cohen, Kamarck, \& Mermelstein, 1983; the Satisfaction with Life Scale, Diener, Emmons, Larsen, \& Griffin, 1985; the Self-Stigma of Mental Illness Scale, Tucker et al., 2013; the Perceived Devaluation Discrimination Scale, Link, 1987; Attitudes Toward Mental Health Treatment, Brown et al., 2010; and the Positive and Negative Affect 
Scale, Watson, Clark, \& Tellegen, 1988). It is beyond the scope of this manuscript to analyze participants' responses to the quantitative measures.

Part Three of the survey consisted of five open-ended questions related to the perceptions of mental health concerns, stigma, seeking support, and counselor attributes. The current study analyzed responses to two open-ended questions in Part Three about perceptions of mental health concerns and stigma. These questions were: (1) What are some of the negative views your culture has about mental illness that might cause stigma? (2) How do you think mental illness stigma applies (or does not apply) to people you know or people in general? In other words, have you known people who have feared seeking mental health treatment because of stigma? The research team deemed these questions as most pertinent in order to explore how African Americans perceive mental health concerns and stigma as opposed to the other three open-ended questions that were related to preferred provider traits and alternative sources of support for mental healthcare

There were 632 respondents to the electronic survey. Inclusion criteria for the study included self-identification as African American as race/ethnic status. The respondents replied to the following multiple choice question: What is your ethnicity? The possible responses were African-American, Asian-American, Hispanic, Multiracial, NativeAmerican, Pacific/Islander, White (Non-Hispanic), and Other (respondents were able to write race/ethic status for Other). The sample analyzed for this manuscript consisted of 210 $(n=210)$ participants who self-identified as African American on Part One of the survey. Respondents who selected an additional race/ethnic status in addition to African American were not included in this study. The primary researcher chose to focus on those who identify as solely African American for this study because gap in the literature and need to 
understand attitudes about mental illness in African American communities. Supplementary participant information is listed in Table 1.

\section{Table 1}

African Americans' Spiritual and Religious Beliefs towards Mental Health

\begin{tabular}{|c|c|c|}
\hline Characteristic & $\mathrm{n}$ & $\%$ \\
\hline \multicolumn{3}{|l|}{ Gender } \\
\hline Female & 159 & 76 \\
\hline Male & 51 & 24 \\
\hline \multicolumn{3}{|l|}{ Age } \\
\hline $18-29$ & 75 & 35.8 \\
\hline $30-39$ & 46 & 22 \\
\hline $40-49$ & 21 & 10 \\
\hline $50-59$ & 40 & 19.1 \\
\hline $60-69$ & 26 & 12.4 \\
\hline $70-79$ & 3 & 1.5 \\
\hline \multicolumn{3}{|l|}{ Marital Status } \\
\hline Single & 121 & 57.6 \\
\hline Cohabitating & 14 & 6.7 \\
\hline Married & 45 & 21.4 \\
\hline Separated/Divorced & 21 & 10 \\
\hline Widowed & 9 & 4.3 \\
\hline \multicolumn{3}{|l|}{ Employment Status } \\
\hline Full Time (30+ Hours) & 77 & 36.7 \\
\hline Part Time (20 Hours or Less) & 22 & 10.5 \\
\hline Unemployed & 69 & 33 \\
\hline Retired & 25 & 11.9 \\
\hline Student & 13 & 6.2 \\
\hline Don't Know/Other & 4 & 1.9 \\
\hline \multicolumn{3}{|l|}{ Insurance Type } \\
\hline Medicare & 46 & 21.9 \\
\hline Medicaid & 56 & 26.7 \\
\hline Tricare & 3 & 1.4 \\
\hline Private Insurance & 60 & 28.6 \\
\hline Uninsured & 29 & 13.8 \\
\hline Unsure/Other & 16 & 5.8 \\
\hline \multicolumn{3}{|l|}{ Community Demographic } \\
\hline Rural & 42 & 20.0 \\
\hline Suburban & 85 & 40.5 \\
\hline Urban & 83 & 39.5 \\
\hline
\end{tabular}

Highest Level of Education 
9th-11th Grade

$15 \quad 7.1$

High School Graduate

$55 \quad 26.2$

Some College Education

$64 \quad 30.5$

Associate's Degree

$30 \quad 14.3$

Bachelor's Degree

$31 \quad 14.8$

Master's Degree

$14 \quad 6.7$

Don't Know

$1 \quad 0.5$

Household Income

Below \$10,000

$\begin{array}{ll}42 & 20.0\end{array}$

\$10-\$19,999

$38 \quad 18.1$

\$20-29,999

$28 \quad 13.3$

$\$ 30-\$ 39,999$

$30 \quad 14.3$

$\$ 40-\$ 49,999$

$13 \quad 6.2$

$\$ 50-\$ 59,999$

$19 \quad 9.0$

Over $\$ 60,000$

$27 \quad 12.9$

Don't Know/Rather Not Say

$13 \quad 6.2$

Spiritual and/or Religious Beliefs

Spiritual and Religious

$115 \quad 54.8$

Spiritual Not Religious

$44 \quad 21.0$

Religious Not Spiritual

22

10.5

Neither Spiritual or Religious

$29 \quad 13.8$

Religious Affiliation

Protestant Christian

$114 \quad 54.3$

Catholic

$14 \quad 6.7$

Muslim

$3 \quad 1.4$

Jewish

$5 \quad 2.4$

Not Religious

Other

$30 \quad 14.3$

Relationships to Mental Health Treatment

Family Has Never Sought Treatment in the Past

$131 \quad 62.4$

Family Has Sought Treatment in the Past

$69 \quad 32.9$

No Friends or Someone They Know Has Sought Treatment

$141 \quad 67.1$

Friend or Someone They Know Has Sought Treatment

$65 \quad 31.0$

Unsure

$14 \quad 6.7$

Personally Never Sought Treatment

$141 \quad 67.1$

Personally Have Sought Treatment

$65 \quad 31.0$

Rather Not Say

$4 \quad 1.9$

Data Analysis 
Recommendations for qualitative content data analysis from Hsieh and Shannon (2005), and Elo and Kyngäs (2008) informed our process. We used an inductive approach to conventional content analysis by studying raw data (i.e., qualitative findings) and making evidenced-based inferences through grouping codes (Elo \& Kyngäs, 2008; Hsieh \& Shannon, 2005). Specifically, we classified large portions of data into smaller content categories based on similarities and cohesion of the data (Finfgeld-Connett, 2014). We used keywords and phrases as the unit of analysis (Insch, Moore, \& Murphy, 1997).

The data analysis process consisted of five steps. First, we (each researcher) immersed ourselves in the data by independently reading all text responses to the two openended survey questions to obtain a sense of the whole. Second, we re-read the text data to identify similarities in participants' responses and highlighted keywords (i.e., codes) and phrases that captured conceptual meanings (i.e., open coding). We made notes of our initial thoughts and opinions by memoing (Finfgeld-Connett, 2014; Hays \& Singh, 2012). During the third step, we sorted keywords and participants' quotes based on the responses to the questions and recorded them in a research codebook. There was one research codebook, which contained code definitions, rules for assigning codes, and specific participant quotes (Krippendorff, 2013). For example, in the research codebook, we defined dangerously violent as when people are feared or perceived as aggressive and unsafe due to having a mental illness. In the fourth step, we collapsed codes to identify emerging themes and placed the codes into meaningful coding categories. As an example, participants' phrases such as "they hurt others" and "they will snap on you" were listed in the violence/dangerous category. In the last step, we applied the coding categories to the entire corpus of text. We repeated the data sorting process until we collectively agreed that the data analyzed were 
captured in six themes that responded to the research questions regarding how African Americans perceive stigma to impact mental health and help-seeking and their attitudes toward mental illness and help-seeking.

\section{Trustworthiness}

We utilized several methods to maximize trustworthiness in the study. First, we immersed ourselves in the literature related to the perceptions of mental illness, stigma, and the African American community. The research team bracketed throughout the research process (Hays \& Singh, 2012). We used memoing by notating participants' common statements to organize thoughts and opinions related to study data to increase dependability (Finfgeld-Connett, 2014). Researcher triangulation (i.e., using the perceptions and skills of multiple researchers in data collection, data analysis, and manuscript writing) was used to enhance the study's rigor and ensure coding validation (Hays \& Singh, 2012). Specifically, we met to code the data, discuss emerging themes, negotiate interpretations of the data, and to reach consensual agreement of the findings (Zhang \& Wildemuth, 2009). We used peer debriefing to acknowledge further any biases or assumptions related to the data. We kept notes of the discussions and reactions to the data in a reflexivity journal (Hays \& Singh, 2012). Finally, we used an external auditor who had training in qualitative research methods. The auditor reviewed the codebook and emerging themes to evaluate the accuracy of the data.

\section{Results}


The following research questions guided the current study, 'How do African Americans perceive stigma to impact mental health and help-seeking? What are the attitudes toward mental illness, and help-seeking within the African American community?" The participants responded to a qualitative survey and the researchers identified six themes: (1) Stigma: Feeling ashamed and being shamed, (2) Violence/danger, (3) Perceptions of ability, (4) Indicator of weakness, (5) Dismissing mental health issues, and (6) Religion and spirituality. These themes represent ideas that the participants believe exist about people with mental illness, how stigma impacts people's decision to seek treatment, and the perceptions of mental health help-seeking that they believe exist in their communities.

\section{Stigma: Feeling Ashamed and Being Shamed}

The most dominate response from participants centered on stigma and feeling ashamed internally and being shamed by others. Participants noted that individuals might be apprehensive about seeking formal mental health services due to perceived negative judgment of others as well as their own internal struggles with being vulnerable in that way. The participants noted that individuals would fear being shamed and ostracized if others know about their mental health experiences. Participants stated that even family and friends could stigmatize and shame their loved ones with mental illness. One participant stated, "They are ashamed to discuss it with anyone. They have been taught to handle their problems themselves without seeking professional help." Some of the participants also spoke to the consequences that resulted from stigma. For example, a participant responded that one negative view that exists is, "People with a mental illness should be kept away 
from people who don't have the illness." Another participant expressed the sentiment this way, "We treated as if we Aliens sometimes."

\section{Violence/Danger}

The idea of violence/danger emerged as a strong theme as many participants stated that people with mental illnesses were seen as violent and a danger to themselves and others. One participant stated, "Be careful around people with mental illness, they may snap on you." The participants spoke about people with mental illness as untrustworthy. One participant even stated, "They are killers". Along these same lines, the perceptions of people with mental illnesses were that they are "crazy." The idea that a person diagnosed with a mental illness is perceived as "crazy" was seen as further evidence that they were dangerous or violent. Another participant added, "You wouldn't trust this person or take them serious and you would be cautious around this person of the hurting you, someone else or themselves."

\section{Perceptions of Ability}

Many participants likened mental illness to intellectual and developmental abilities as mental illness was perceived to correlate with one's mental capabilities. In other words, people with mental health struggles were less intelligent and differently abled in their development. For example, one participant stated, "A lot of people think that people with mental illnesses might be stupid or 'out of their mind'." Further, participants noted that the 
perception of people with mental illnesses is that they are incompetent and intellectually inferior to others without a diagnosis. The participants did not differentiate between emotional health diagnosis (e.g., depression) and cognitive related diagnoses (e.g., autism). Another participant added that the perception of individuals with mental illness is that, "Mental health people can't be smart or reliable and get in the way all the time." As a result, people with mental illnesses were considered disposable or a burden because of their perceived lack of intelligence. Further, several participants used derogatory remarks (e.g., "retarded") to describe the perceptions that exist about people with mental illness.

\section{Dismissing Mental Health Issues}

The participants in the current study noted the tendency of African Americans to dismiss or minimize the prevalence of mental health issues. The perception is that individuals with a mental illness exaggerate their symptomatology and its impact. One participant responded, "Mental illness is almost a forbidden subject in the Black/African American culture. We tend to believe in holding things in or handling them through hostile acts as a coping mechanism. People in my culture say things like 'shrinks are for White people' and 'it doesn't work effectively'". Another participant added, "People believe that mental illness is just a easy way out. I, however, believe that life experiences may be contributed to your mental capacity."

\section{Indicator of Weakness}


Participants noted the perception that mental illness "makes you weak or less than a person." One participant noted, "Some African Americans feel that seeking help for mental illness is admitting weakness of some sort, especially older individuals." Another stated, "Black people don't deal with mental illness because we are considered strong." Thus, the ideas of weakness have implications for perceptions of mental illness as well as help seeking behaviors. The participants stated that individuals might not seek help for mental health concerns because of fear of being considered weak for seeming to lack adequate and acceptable coping strategies. Intersections of identity came up for many of the participants as they reflected on the role of gender norms in conceptualizations of strength vs. weakness. Specifically, they indicated that African American men with mental illness were seen as especially weak. "Black people oftentimes do not seek counseling especially our black men. To them, it is a sign of frailty or weakness. Recently, those statistics are changing in the positive, but in my opinion, they are not changing fast enough". Another stated, "Needing help is weak, especially if you are a man."

\section{Religion and Spirituality}

A few participants noted the tendency of African Americans to rely on religious and spiritual coping practices when dealing with mental illness. Further, the participants provided insight into the notion that to have a mental illness would be considered counterreligious. In other words, a person identified as having a mental illness may be considered to be lacking in faith, and even more so, to not be religious at all. When asked about the perceptions of help-seeking, one participant responded, "...blasphemous because blacks 
should rely on Jesus to solve our problems". Another participant stated, "Black people do not go for mental help. Just pray and ask God to show you the way."

\section{Discussion}

In the current study, 210 African Americans responded to a survey on their beliefs about the negative perceptions of mental illness that exist in their communities. The findings suggest that negative perceptions persist in African American communities regarding individuals with mental illness (Fripp \& Carlson, 2017). This discussion section will situate these current findings within the existing empirical landscape in order to see how these results compare to existing findings and how they may also offer new knowledge.

Stigma exists when individuals fear that they will be isolated, ridiculed, or mistreated because of an aspect of their identity (Crowe et al., 2016). For many individuals, this fear keeps them from seeking help for mental health related concerns because they do not want to be labeled and experience the aforementioned societal consequences. A number of the negative perceptions expressed by participants in the current study align with stigmatized beliefs about people with mental illness across populations (Crowe et al., 2016). However, given the pre-existing marginalization and oppression that many African Americans face because of their racial identities, it is essential to unpack how stigma related to mental illness may uniquely impact their experience. Many participants identified beliefs about mental illness that were very similar to stereotypes related to race, ethnicity, and gender for African Americans (Welch, 2007). 
Fear and danger related to individuals with mental illness in African American communities emerged as a theme. The prevailing theme of people with mental illnesses as dangerous and violent is consistent with existing literature (Anglin et al., 2006; Rao et al., 2007). Historically, individuals with a mental illness have been "othered". .Scholars (e.g., Keating \& Robertson, 2004) have explored how this fear may be complex and two-fold. In other words, African Americans fear mental health systems and mental health professionals fear consumers. Again, although fear of seeking treatment may exist across populations, the projection of dangerousness by mental health professionals on consumers may be greater and nuanced for African Americans. Keating and Robertson (2004) found that participants would be treated similarly in mental health agencies as they were by other organizations such as police and the criminal justice system. In particular, Black men may be subjected to harsher stereotyping. It is important to unpack how societal conception of African American male masculinity may have impacted the results. Ideas about the presentation of Black men date back to slavery as African American masculinity was very narrowly defined (Ward, 2005). These negative perceptions, along with the intersections of racism, may help explain the underutilization of service within African American communities.

In sum, the findings in this study support the long-standing notion that mental health stigma exists in African Americans communities as perceptions of mental illness tend to be negative and deficit based. More specifically, seeking help may be considered a sign of weakness or a lack of intelligence. Avent Harris and Wong (2018) found that it is important to pay attention to the timing of African Americans' help-seeking as well as participants in that study referenced counseling as a last resort. The findings of the current 
study provide some context for why African Americans may be reluctant to seek help until symptoms have progressed so severe.

The findings do provide some insight on how perceptions of individual illness may reflect stereotypes that exist about African Americans (e.g., violence, dangerousness). This study is also important as it illuminates the participants' own words in describing the perceptions that exist about mental illness.

\section{Implications for Counselors}

Counselors have a unique opportunity to increase mental health literacy in African American communities to help mitigate these challenges. Kutcher, Wei, and Coniglio (2016, p.155) define mental health literacy as "understanding how to obtain and maintain positive mental health; understanding mental disorders and their treatments; decreasing stigma related to mental disorders; and, enhancing help-seeking efficacy." Counselors who attend to the different components increasing individuals' literacy may decrease shame related to mental health treatment. First, counselors could be intentional in promoting the language of health rather than illness as it relates to emotional and mental well-being. This more strengths-based language may decrease individuals propensity to internalize shame and others' inclination to shame or isolate those who have received a diagnosis. Additionally, clinicians could connect clients with other African Americans who have a mental illness and are functioning well to offer a counter-narrative of the negative stereotypes that may exist. 
The participants in the current study spoke to the fact that the mental illness was often minimized in their communities. Therefore, education, awareness, and advocacy efforts should be approached from a macro-level. In other words, it is not enough if one person feels comfortable seeking professional counseling services if members of their own communities would then dismiss them. Mental health awareness month (i.e., May) offers a great platform for educational programming (NAMI, 2018).

In the current study, participants noted that the perception that exists in African American communities is that individuals who are dealing with mental health issues should rely on God for their primary coping. There are a number of ways in which mental health professionals can serve as leaders in the integration of mental health and spirituality. First, collaboration, especially with local pastors, will be vital (Avent et al., 2015; Dempsey et al., 2016). This sentiment is particularly true for African Americans, as pastors serve as leaders in spiritual, economic, and social matters (Avent et al., 2015; Lincoln \& Mamiya, 1990). Pastors and counselors could co-host educational summits so that individuals see these two professions working together. Clinicians could identify or create publications and materials that marry religious beliefs and education about mental health. Counselors can build relationships with pastors so that they can provide accountability on areas of mental health. For instance, clinicians could encourage pastors to be affirming in their sermons, encourage self-care and counseling from the pulpit, and refer parishioners who need services (Ratts, Singh, Nassar-McMilian, Butler, \& McCullough, 2016).

The current findings also provide some insight into relevant theoretical frameworks for counselors to integrate in their work. For instance, CRT (Bell, 1995) offers a lens to understand better the role of race and racism in African American's perceptions of the 
negative perceptions of individuals who are diagnosed with mental illness. At present, CRT is not widely discussed in counselor education training programs (Haskins \& Singh, 2015). However, by considering the notion that racism will always exist and permeates every fabric of society is important for counselors to inform ideas about stigma and services for the African American clients who do decide to seek treatment. Counselors must consider how their clients who are African American and who do have a mental health diagnosis may face greater discrimination. Also, practitioners must consider how these negative stereotypes have been internalized and therefore, perpetuated in African American communities.

While previous researchers (Avent Harris \& Wong, 2018; Fripp \& Carlson, 2017) explored how stigma exists in African American communities it may be more limited in how negative perceptions of mental illness parallel with ideas of Blackness and the compounding impact that may have on clients. The subtlety in which systemic racism and mental health stigma intersect for African Americans, and even more for African American men, may cause these ideas to go unaddressed. CRT provides a necessary framework to grapple with these complexities (Trahan \& Lemberger, 2014). Clients who face discrimination and isolation in broader social contexts because of their race may be reluctant to then identify with a mental illness for fear they may lose what power and privilege they may hold within their racial/ethnic communities (Brown, 2008). Mental health professionals can use narrative storytelling to invite clients to discuss how mental health and race intersect for them and how this impacts their position within their communities and the greater society at large. Also, if clinicians are to truly ascribe to CRT 
and the Multicultural and Social Justice competencies (Ratts et al., 2016), they must reflect on how these revelations may work to inform meaningful change on a policy level.

\section{Limitations and Opportunities for Future Research}

This study has limitations to consider when interpreting the results. First, the researchers' were not able to follow-up with participants for additional comments or questions. Second, it is possible that participants were apprehensive about disclosing indepth mental health information due to mental illness stigma. Additional opportunities to build trust and rapport in the researcher-participant relationship may have enhanced the findings of the study. Qualitative research is intended to be exploratory in nature (Hays \& Singh, 2012). As such, there is the possibility that some of the meaning in the data could be lost through the data reduction techniques employed in the methodological procedures and processes.

It is also important to consider how the participants' demographics may limit the interpretation of the findings. The sample was overwhelmingly female (76\%). Thus, the findings could potentially reflect a more African American female perspective. Future researchers could focus more specifically on African American males' perceptions of stigma and mental illness in their communities. Future researchers need to also be intentional in recruiting a diverse sample that may be more representative of this population.

Researchers interested in African American mental health could expand the current study by conducting focus groups in which African Americans discussed their perceptions of mental illness and help-seeking. This type of study would add to the literature by 
understanding whether these perceptions maintain in-group interactions. This understanding could inform counselors about the potential benefits or risks integrating group counseling into African Americans' treatment plans. Additionally, the findings of the study indicated that African Americans rely on their spirituality and faith-based communities to address mental health issues. Researchers could consider various research designs, such as participatory action research, that can include community representatives to enhance scholarly discourse by including multiple viewpoints and experiences with the population and topic area. It is also important to note that the survey question referenced "your culture". Although, all of the participants identified as African American/Black the answers provided may have referred to another aspect of "culture" as culture is not regulated solely to race/ethnicity. This consideration provides an opportunity for future research to delve more deeply into the aspect of culture.

\section{Conclusion}

In conclusion, the current study both reaffirms existing ideas about the negative stigma that exists about individuals with mental illness. However, the results offer additional perspective on how the intersection of identity may impact African Americans' ideas about mental illness and their rationale for decisions regarding help-seeking. The participants in this study identified ideas of danger, violence, stigma, and religious preference as perceptions within African American communities about mental health and treatment. 


\section{References}

Anglin, D. M., Link, B. G., \& Phelan, J. C. (2006). Racial differences in stigmatizing attitudes toward people with mental illness. Psychiatric Services, 57, 857-862.

Avent, J. R., Cashwell, C. S., \& Brown-Jeffy, S. (2015). African American pastors on mental health, coping, and help seeking. Counseling and Values, 60(1), 32-47.

Avent Harris, J. R., \& Wong, C. D. (2018). African American College Students, the Black Church, and Counseling. Journal of College Counseling, 21(1), 15-28.

Banyard, V. L., \& Miller, K. E. (1998). The powerful potential of qualitative research for community psychology. American Journal of Community Psychology, 26(4), 485505.

Bell, D. A. (1995). Who's afraid of critical race theory? University of Illinois Law Review, 1995(4), 893.

Brown, C., Conner, K. O., Copeland, V. C., Grote, N., Beach, S., Battista, D., \& Reynolds, C. F. (2010). Depression stigma, race, and treatment seeking behavior and attitudes. Journal of Community Psychology, 38(3), 350-368. doi:

10.1002/jcop.20368

Brown, T. N. (2008). Race, racism, and mental health: Elaboration of critical race theory's contribution to the sociology of mental health. Contemporary Justice Review, 11(1), 53-62.

Centers for Disease Control and Prevention, US Department of Health and Human Services. (2016). Health, United States, 2015: With special feature on racial and 
ethnic health disparities [DHHS Publication No. 2016-1232]. Retrieved from https://www.cdc.gov/nchs/data/hus/hus15.pdf.

Chatters, L. M., Taylor, R. J., Lincoln, K. D., \& Schroepfer, T. (2002). Patterns of informal support from family and church members among African Americans. Journal of Black Studies, 33(1), 66-85.

Cohen, S., Kamarck, T., \& Mermelstein, R. (1983). A global measure of perceived stress. Journal of Health and Social Behavior, 24(4), 385-396.

Creswell, J. W., \& Creswell, J. D. (2018). Research design: Qualitative, quantitative, and mixed methods approaches ( $5^{\text {th }}$ ed.). Thousand Oaks, CA: Sage.

Crowe, A., Averett, P., Scott Glass, J., Dotson-Blake, K. P., Grissom, S. E., Ficken, D. K., \& Holmes, J. A. (2016). Mental health stigma: Personal and cultural impacts on attitudes. Journal of Counselor Practice, 7(2), 97-119.

Dempsey, K., Butler, S. K., \& Gaither, L. (2016). Black churches and mental health professionals: Can this collaboration work?. Journal of Black Studies, 47(1), 73 87.

Elo, S., \& Kyngas, H. (2007). The qualitative content analysis process. Journal of Advanced Nursing, 62(1), 107-115. doi:10.1111/j.1365-2648.2007.04569.x

Finfgeld-Connett, D. (2014). Use of content analysis to conduct knowledge-building and theory-generating qualitative systematic reviews. Qualitative Research, 14(3), 341-352.

Fripp, J. A., \& Carlson, R. G. (2017). Exploring the influence of attitude and stigma on participation of African American and Latino populations in mental health services. Journal of Multicultural Counseling and Development, 45(2), 80-94. 
Gary, F. A. (2009). Stigma: barrier to mental health care among ethnic minorities. Issues in Mental Health Nursing, 26(10), 979-999. https://doi.org/10.1080/01612840500280638

Hall, J. C. (2018). It is tough being a Black woman: Intergenerational stress and coping. Journal of Black Studies, 49(5), 481-501.

Haskins, N. H., \& Singh, A. (2015). Critical race theory and counselor education pedagogy: Creating equitable training. Counselor Education and Supervision, 54(4), 288-301.

Hays, D. G., \& Singh, A. A. (2012). Qualitative inquiry in clinical and educational settings. New York, NY: Guilford Press.

Hays, K., \& Lincoln, K. D. (2017). Mental health help-seeking profiles among African Americans: Exploring the influence of religion. Race and Social Problems, 9(2), $127-138$.

Hsieh, H., \& Shannon, S. E. (2005). Three approaches to qualitative content analysis. Qualitative Health Research, 15(9), 1277-1288. doi:10.1177/1049732305276687

Insch, G. S., Moore, J. E., \& Murphy, L. D. (1997). Content analysis in leadership research: Examples, procedures, and suggestions for future use. The Leadership Quarterly, 8(1), 1-25.

Keating, F., \& Robertson, D. (2004). Fear, black people and mental illness: A vicious circle? Health and Social Care in the Community, 12(5), 439-447.

Krippendorff, K. (2013). Content analysis: An introduction to its methodology $\left(3^{\text {rd }}\right.$ ed.). Los Angeles, CA: SAGE. 
Kutcher, S., Wei, Y., \& Coniglio, C. (2016). Mental Health Literacy: Past, Present, and Future. The Canadian Journal of Psychiatry, 61(3), 154158. https://doi.org/10.1177/0706743715616609

Lincoln, C. E., \& Mamiya, L. H. (1990). The Black church in the African American experience. Durham, NC: Duke University Press.

Link, B. G. (1987). Understanding labeling effects in the area of mental disorders: An assessment of the effects of expectations of rejection. American Sociological Review, 52(1), 96-112. doi: 10.2307/2095395

Matthews, A. K., Corrigan, P. W., Smith, B. M., \& Aranda, F. (2006). A qualitative exploration of African-Americans' attitudes toward mental illness and mental illness treatment seeking. Rehabilitation Education, 20, 253-268.

National Alliance on Mental Illness. (2018). African American mental health. Retrieved from https://www.nami.org/Find-Support/Diverse-Communities/AfricanAmericans.

Office of Minority Mental Health, US Department of Health and Human Services. (2017). Mental health and African Americans. Retrieved from https://minorityhealth.hhs.gov/omh/browse.aspx?lvl=4\&lvlid=24.

Rao, D., Feinglass, J., \& Corrigan, P. (2007). Racial and ethnic disparities in mental illness stigma. The Journal of Nervous and Mental Disease, 195, 1020-1023.

Ratts, M. J., Singh, A. A., Nassar-McMillan, S., Butler, S. K., \& McCullough, J. R. (2016). Multicultural and social justice counseling competencies: Guidelines for the counseling profession. Journal of Multicultural Counseling and Development, 44(1), 28-48. 
Sanders Thompson, V. L., Brazile, A., \& Akbar, M. (2004). African Americans' perceptions of psychotherapy and psychotherapists. Professional Psychology: Research and Practice, 35, 19-26.

Substance Abuse and Mental Health Services Administration. (2015). Racial/ethnic differences in mental health service use among adults. Retrieved from https://www.integration.samhsa.gov/MHServicesUseAmongAdults.pdf

Trahan, D. P., \& Lemberger, M. E. (2014). Critical race theory as a decisional framework for the ethical counseling of African American clients. Counseling and Values, 59(1), 112-124.

Tucker, J., Hammer, J. H., Vogel, D. L., Bitman-Heinrichs, R., Wade, N.G., \& Maier, E. J. (2013). Disentangling self-stigma: Are mental illness and help-seeking selfstigmas different? Journal of Counseling Psychology, 60(4), 520-531. doi: $10.1037 / \mathrm{a} 0033555$

Ward, E. G. (2005). Homophobia, hypermasculinity and the US black church. Culture, health \& sexuality, 7(5), 493-504.

Watson, D., Clark, L. A., \& Tellegen, A. (1988). Development and validation of brief measures of positive and negative affect: The PANAS scales. Journal of Personality and Social Psychology, 54(6), 1063-1070. http://dx.doi.org/10.1037/0022-3514.54.6.1063

Welch, K. (2007). Black criminal stereotypes and racial profiling. Journal of Contemporary Criminal Justice, 23(3), 276-288. https://doi.org/10.1177/1043986207306870 
Zhang, Y., \& Wildemuth, B. M. (2009). Qualitative analysis of content. In B. Wildemuth (Ed.), Applications of social research methods to questions in information and library science (pp 308-319). Westport, CT: Libraries Unlimited. 